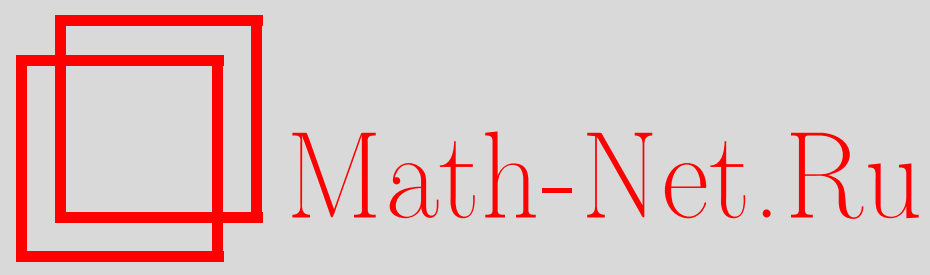

С. Я. Новиков, Об особенностях оператора вложения симметричных функциональных пространств на [0,1], Maтем. заметки, 1997, том 62, выпуск 4, 549-563

DOI: https://doi.org/10.4213/mzm1638

Использование Общероссийского математического портала Math-Net.Ru подразумевает, что вы прочитали и согласны с пользовательским соглашением http://www.mathnet.ru/rus/agreement

Параметры загрузки:

IP: 52.6 .47 .48

26 апреля 2023 г., 12:30:19

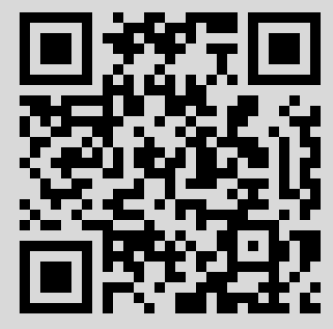




\section{ОБ ОСОБЕННОСТЯХ ОПЕРАТОРА ВЛОЖЕНИЯ СИММЕТРИЧНЫХ ФУНКЦИОНАЛЬНЫХ ПРОСТРАНСТВ НА $[0,1]$}

\section{С. Я. Новиков}

Исследованы такие свойства оператора вложения $I\left(X_{1}, X_{2}\right), X_{1} \subset X_{2}$, между симметричными функциональными пространствами на $[0,1]$, как слабая компактность, строгая сингулярность (в двух вариантах), абсолютная суммируемость. Рассматриваются банаховы и квазибанаховы пространства. Получено полное описание замкнутой по мере линейной оболочки последовательности $\left(g_{n}^{(r)}\right)$ независимых симметричных одинаково распределенных случайных величин с

$$
d\left(g_{n}^{(r)} ; t\right)=\operatorname{meas}\left(\omega:\left|g_{n}^{(r)}(\omega)\right|>t\right)=\frac{1}{t^{r}}, \quad t \geqslant 1, \quad 0<r<\infty
$$

и найдены границы для этого подпространства в шкале симметричных пространств.

Библиография: 19 названий.

Введение. Основной объект, который мы рассматриваем в данной работе,- -это оператор тождественного вложения $I\left(X_{1}, X_{2}\right)$, действующий между симметричными функциональными пространствами $X_{1}$ и $X_{2}$, в ситуации, когда $X_{1} \subset X_{2}$. В п. 2 исследован оператор $I\left(L^{\infty}, X\right)$ для банахова симметричного пространства $X$. Доказано, что этот оператор слабо компактен и строго сингулярен для любого $X \neq L^{\infty}$. Найдены необходимые и достаточные условия $p$ - и $(q, p)$-суммируемости этого оператора. Выясняется важная роль свойства Данфорда-Петтиса в этом круге вопросов.

Пункт 3 посвящен в основном оператору $I\left(X, L^{1}\right)$ для банахова симметричного пространства $X$. Здесь вводится понятие дизъюнктной строгой сингулярности оператора и доказьвается, что рассматриваемьй оператор является дизъюнктно строго сингулярным для любого $X \neq L^{1}$.

В п. 4 мы переходим к рассмотрению квазибанаховых пространств. Показано, что при выполнении дополнительного условия $\gamma$-вьпуклости имеют место аналоги теорем предыдуших пунктов. Здесь же построены так назьваемые "сквозные" подпространства, порожденные последовательностями независимых одинаково распределенных функций.

Некоторые результаты этой работы докладьвались автором на Международной конференции, посвященной 90-летию академика С. М. Никольского.

1. Обозначения. Теория банаховых симметричных функциональных пространств на русском языке достаточно полно изложена в [1]. Последние годы наметилась тенденция включать в класс симметричных пространств и квазибанаховы пространства. Мы также будем рассматривать квазибанаховы пространства в данной работе. 
Обозначим через $L_{0}$ пространство всех вещественных измеримых функций на $[0,1]$; через $\varkappa_{A}-$ характеристическую функцию измеримого по Лебегу множества $A$; через meas $A$ или $|A|$ - меру Лебега множества $A$; через $f^{*}$ - убывающую перестановку функции $|f|$. Квазибанахово симметричное пространство (сокращенно КБСП) - это полное квазинормированное векторное пространство $(X,\|\cdot\|)$ такое, что $X \subset L_{0}$, $\left\|\varkappa_{A}\right\|=1$, если meas $A=1$, и справедлива импликация:

$$
g \in X, f \in L_{0}, f^{*} \leqslant g^{*} \Longrightarrow f \in X \text { и }\|f\| \leqslant\|g\| .
$$

Квазинорма $\|\cdot\|$ является функционалом, которьй удовлетворяет всем аксиомам нормы, кроме неравенства треугольника, которое заменяется более слабым неравенством

$$
\|x+y\| \leqslant K(\|x\|+\|y\|), \quad x, y \in X,
$$

с некоторой $K>1$.

В дальнейшем слово “оператор” будет означать ограниченньй линейньй оператор. Классические лебеговы пространства обозначаются стандартными символами $L^{p}$.

2. Граничные пространства для оператора вложения $I\left(L^{\infty}, X\right)$. Если между двумя СП $X_{1}$ и $X_{2}$ имеет место включение $X_{1} \subset X_{2}$, товозникает оператор $I: f \in X_{1}$ $\rightarrow f \in X_{2}$, которьй обозначим через $I\left(X_{1}, X_{2}\right)$.

Лемма 2.1. Пусть $X-$ банахово или квазибанахово СП. Пространство $L^{\infty}$ непрерывно вложсено в $X$, и выполняется неравенство

$$
\|f\|_{X} \leqslant\|f\|_{\infty}, \quad f \in L^{\infty} .
$$

ДокАЗАТЕльство. Пусть $f \in L^{\infty}$ и $f \geqslant 0$. Тогда

$$
f^{*}(0)=\|f\|_{\infty}<\infty, \quad f^{*}(t) \leqslant f^{*}(0) \varkappa_{[0,1]}(t), \quad t \in[0,1],
$$

и $\|f\|_{X} \leqslant f^{*}(0)=\|f\|_{\infty}$.

Теорема 2.2. Если $X$-банахово СП, $X \neq L^{\infty}$, то оператор вложсения $I\left(L^{\infty}, X\right)$ слабо компактен.

ДокАЗАТЕльство. Мы покажем, что рассматриваемьй оператор вложения можно факторизовать через рефлексивное банахово СП. Пусть $\varphi_{X}(t):=\left\|\varkappa_{[0, t]}\right\|_{X}$ обозначает фундаментальную функцию пространства $X$, которую, не ограничивая общности, можно считать вогнутой. Построим пространство Лоренца $\Lambda\left(\varphi_{X}\right)$ с нормой

$$
\|f\|_{\Lambda\left(\varphi_{X}\right)}=\int_{0}^{1} f^{*}(t) d \varphi_{X}(t) .
$$

Это пространство слабо секвенциально полно и непрерьвно вложено в $X$ [1]. Далее, построим $r$-степенное преобразование пространства $\Lambda\left(\varphi_{X}\right)$ для некоторого $r>1$, которое обозначим через $\Lambda^{r}\left(\varphi_{X}\right)$. Напомним, что

$$
\Lambda^{r}\left(\varphi_{X}\right)=\left\{g:|g|^{r} \in \Lambda\left(\varphi_{X}\right)\right\}, \quad\|g\|_{\Lambda^{r}\left(\varphi_{X}\right)}=\left\||g|^{r}\right\|_{\Lambda\left(\varphi_{X}\right)}^{1 / r} .
$$

Пространство $\Lambda^{r}\left(\varphi_{X}\right)$ рефлексивно [2] и непрерывно вложено в $\Lambda\left(\varphi_{X}\right)$. Итак, мы получили цепочку непрерьвных вложений $L^{\infty} \subset \Lambda^{r}\left(\varphi_{X}\right) \subset \Lambda\left(\varphi_{X}\right) \subset X$, один элемент которой - рефлексивное пространство. 
СледСтвиЕ 2.3. Для каждого банахова СП $X, X \neq L^{\infty}, X \neq L^{1}$, существуют "рефлексивные ворота", т.е. пара банаховых рефлексивных СП $X_{\sim}$ u $X^{\sim}$ таких, что $X_{\sim} \subset X \subset X^{\sim}$.

ДокаЗАТЕЛЬСтво. Построение пространства $X_{\sim}$ описано выше. Для построения $X \sim$ воспользуемся соображениями двойственности. Введем пространство, ассоциированное к $X$, и обозначим его $X^{\prime}$ (см. [1]). Из общей теории банаховых идеальных пространств следует, что ассоциированное к рефлексивному СП само является рефлексивным (см., например, [3, следствие из теоремы 1.5]). Найдем рефлексивное СП $X_{\sim}^{\prime} \subset X^{\prime}$. Отсюда $\left(X_{\sim}^{\prime}\right)^{\prime} \supset X^{\prime \prime} \supset X$, и остается положить $X^{\sim}=\left(X_{\sim}^{\prime}\right)^{\prime}$.

Напомним, что банахово пространство $X$ обладает свойством Данфорда-Петтиса, если для любого банахова пространства $Y$, для любого слабо компактного оператора $T: X \rightarrow Y$ и для любого выпуклого уравновешенного слабо компактного множества $K \subset X$ множество $T(K)$ компактно в сильной топологии пространства $Y[4$, гл. 9]. В дальнейшем, желая показать, что пространство $X$ обладает свойством Данфорда-Петтиса, мы используем запись: $X \in \mathrm{DP}$. Известно много эквивалентных переформулировок свойства Данфорда-Петтиса. Для дальнейшего нам понадобится следующее требование: если $T: X \rightarrow Y$ слабо компактен и последовательность $x_{i} \rightarrow 0$ слабо в $X$, то $\left\|T x_{i}\right\|_{Y} \rightarrow 0$ [5, теорема 5]. Оператор, который переводит слабо сходящиеся последовательности в сильно сходящиеся, назьвают оператором Данфорда-Петтиса.

Теоремы о том, что пространства $L^{\infty} \in \mathrm{DP}$ и $L^{1} \in \mathrm{DP}$, являются классическими $[4$, гл. 9]. Оказывается, что в классе банаховых СП других пространств с этим свойством нет.

СлЕДСтвиЕ 2.4. В классе банаховых СП только два пространства: $L^{1}$ и $L^{\infty}$,имеют свойство Данфорда-Петтиса.

ДоказАтЕльство. Предположим существование банахова СП $X \neq L^{1}, X \neq L^{\infty}$, обладающего свойством Данфорда-Петтиса. Используя следствие 2.3 , строим "рефлексивные ворота" $X_{\sim}, X^{\sim}$ для пространства $X$. Пусть $x_{n}(t)=\sin (2 \pi n t), n=1,2, \ldots$. Эта последовательность слабо сходится к 0 в пространстве $X_{\sim}$. Действительно, в силу его рефлексивности банахово сопряженное $X_{\sim}^{*}=X_{\sim}^{\prime} \subset L^{1}$ и слабая сходимость обосновывается классической леммой Римана о коэффициентах тригонометрического ряда. Из непрерывности оператора $I\left(X_{\sim}, X\right)$ следует слабая сходимость последовательности $\left\{x_{n}\right\}$ к 0 и в пространстве $X$. Из нашего предположения и рефлексивности пространства $X^{\sim}$ получаем сходимость $\left\|x_{n}\right\|_{X} \sim 0, n \rightarrow \infty$. Из вложения $X^{\sim} \subset L^{1}[1]$ следует сходимость $\left\|x_{n}\right\|_{L^{1}} \rightarrow 0, n \rightarrow \infty$. Но это, очевидно, не так.

СледСтвие 2.5. Если $X-\mathrm{C \Pi}, X \neq L^{1}, X \neq L^{\infty}$, то ни в пространстве $L^{1}$, ни в пространстве $L^{\infty}$ не существует дополняемого подпространства, изоморфно$20 X$.

ДокАЗАТЕЛЬСтво. Достаточно применить известный результат о том, что свойство Данфорда-Петтиса наследуется дополняемыми подпространствами [4], и следствие 2.4.

Перейдем к рассмотрению строгой сингулярности оператора $I\left(L^{\infty}, X\right)$. Пусть $X$ и $Y$ - банаховы или квазибанаховы пространства. Оператор $T: X \rightarrow Y$ называется $c m p o-$ го сингулярны., если сужение $T$ на произвольное бесконечномерное подпространство 
пространства $X$ не является изоморфизмом. Множество всех строго сингулярных операторов так же, как и множество всех слабо компактных операторов, образует операторный идеал $[6,1.5 .2$ и 1.9.4].

Теорема 2.6. Если $X$ - банахово СП, $X \neq L^{\infty}$, то $I\left(L^{\infty}, X\right)$ строго сингулярен. Это означает, что в $X$ не существует бесконечномерного подпространства, состоящего только из ограниченных функиий.

ДокАЗАТЕЛЬСТво. Пусть $H$ - (замкнутое) подпространство пространства $X$ и $H \subset L^{\infty}$. Как и вьше, находим рефлексивное $X_{\sim}$ такое, что $L^{\infty} \subset X_{\sim} \subset X$. Нормы этих трех пространств эквивалентны на $H$. Следовательно, единичный шар $H$ слабо компактен в $L^{\infty}$. Но пространство $L^{\infty} \in \mathrm{DP}$, и $I\left(L^{\infty}, X\right)$ слабо компактен (теорема 2.2). Поэтому единичный шар $H$ сильно компактен в $X$, т.е. $H$ конечномерно.

Теорема 2.6 является обобщением теоремы А. Гротендика о строгой сингулярности $I\left(L^{\infty}, L^{p}\right)$ для $0<p<\infty[7$, гл. 5].

Рассмотрим вопрос об абсолютной суммируемости оператора $I\left(L^{\infty}, X\right)$.

ОПРЕДЕЛЕНИЕ 2.7. Пусть $X$ - банахово, $Y$ - квазибанахово пространство, $0<p \leqslant$ $q<\infty$. Оператор $T: X \rightarrow Y$ назьвается $(q, p)$-абсолютно суммирующим (сокращенно $\left.T \in \Pi_{q, p}(X, Y)\right)$, если найдется такая постоянная $C>0$, что для любого $n$ и произвольных элементов $x_{1}, \ldots, x_{n} \in X$ вьполняется неравенство

$$
\left(\sum_{i=1}^{n}\left\|T x_{i}\right\|^{q}\right)^{1 / q} \leqslant C \sup \left\{\left(\sum_{i=1}^{n}\left|F\left(x_{i}\right)\right|^{p}\right)^{1 / p}:\|F\|_{X^{*}} \leqslant 1\right\}
$$

Наименьшее возможное значение постоянной $C$ обозначается через $\pi_{q, p}(T)$. Слово “абсолютно" в дальнейшем иногда опускается.

Определение содержательно лишш при $p \leqslant q$; если $p>q$, то лишь нулевой оператор может быть $(q, p)$-абсолютно суммируюшим. Для $p=q$ употребляются обозначения $\Pi_{p}$ и $\pi_{p}$ (подробнее см. [3], [6], [8], [9]). Заметим только, что если $X=C(K), K$ - метрический компакт, или $X=L^{\infty}$, то неравенство (1) при $p=1$ может быть записано в форме

$$
\left(\sum_{i=1}^{n}\left\|T x_{i}\right\|^{q}\right)^{1 / q} \leqslant C\left\|\sum_{i=1}^{n}\left|x_{i}\right|\right\|_{X}
$$

Упомянутая выше теорема Гротендика в сочетании с теоремой А. Пича о факторизации $p$-суммирующих операторов (см., например, $[9,1.3 .4])$ дает сравнительно простой способ доказательства строгой сингулярности любого $p$-суммирующего оператора, $0<p<\infty$. Другой способ рассуждений представлен в $[6,4.6 .14,17.2 .4,17.2 .7]$.

Теорема 2.7. Пусть БСП $X_{1}$ вложсено в БСП $X_{2}, p \geqslant 1$. Тогда

$$
I\left(X_{1}, X_{2}\right) \in \Pi_{p} \Longleftrightarrow X_{1}=L^{\infty}, X_{2} \supseteq L^{p} .
$$


ДокАЗАТЕЛЬСТво. $(\Longrightarrow)$ Всякий $p$-суммирующий оператор является оператором Данфорда-Петтиса $[9,1.3 .6]$. Это обстоятельство позволяет нам повторить рассуждения при доказательстве следствия 2.4, с помошью которых получаем, что $X_{1}=L^{\infty}$. По следствию из теоремы Пича $[9,1.3 .5]$ при $p \geqslant 1$ сушествует вероятностная мера $\nu$ на $[0,1]$ такая, что

$$
\|f\|_{X_{2}} \leqslant \pi_{p}(I)\left(\int|f(s)|^{p} d \nu(s)\right)^{1 / p}, \quad f \in C[0,1] .
$$

Для $t \in[0,1]$ определим $f_{t}(s):=f(t+s)$, сложение по mod1. Учитьвая симметрию пространства $X_{2}$, получаем

$$
\|f\|_{X_{2}}^{p}=\left\|f_{t}\right\|_{X_{2}}^{p} \leqslant \pi_{p}^{p}(I) \int\left|f_{t}\right|^{p} d \nu(s), \quad t \in[0,1]
$$

Последнее неравенство интегрируем по лебеговой мере на $[0,1]$

$$
\begin{aligned}
\|f\|_{X_{2}}^{p} & =\int_{0}^{1}\left\|f_{t}\right\|_{X_{2}}^{p} d t \leqslant \pi_{p}^{p}(I) \int_{0}^{1} \int\left|f_{t}(s)\right|^{p} d \nu(s) d t \\
& =\pi_{p}^{p}(I) \iint_{0}^{1}|f(t)|^{p} d t d \nu(s)=\pi_{p}^{p}(I)\|f\|_{L^{p}}^{p}, \quad f \in C[0,1] .
\end{aligned}
$$

Из полученного неравенства следует включение $X_{2} \supseteq L^{p}$.

$(\Longleftarrow)$ Оператор $I\left(L^{\infty}, L^{p}\right) \in \Pi_{p}$, и множество $\Pi_{p}$ - операторный идеал (см., например, [9]).

Теорема 2.7 полностью решает вопрос о $p$-суммируемости, $p \geqslant 1$, оператора вложения между произвольными БСП, а не только между $L^{\infty}$ и $X$. Для $(q, p)$-суммируемости с $p<q$ полное решение, видимо, неизвестно. Тем не менее для оператора, которому посвящен этот пункт, решение все же есть.

Teорема 2.8. Пусть $X-$ БСП, $1 \leqslant p<q<\infty$. Тогда

$$
I\left(L^{\infty}, X\right) \in \Pi_{q, p} \Longleftrightarrow X \supseteq L^{q, 1}
$$

әде

$$
\begin{gathered}
L^{q, 1}:=\left\{f \in L_{0}:\|f\|_{q, 1}<\infty\right\} \\
\|f\|_{q, 1}=\frac{1}{q} \int_{0}^{1} f^{*}(t) t^{1 / q} \frac{d t}{t}=\int_{0}^{1}(\operatorname{meas}(s:|f(s)|>t))^{1 / q} d t .
\end{gathered}
$$

(Заметим, что $L^{q, 1}=\Lambda\left(\varphi_{q}\right), \varphi_{q}(t)=t^{1 / q}$, см. теорему 2.2.) 
ДокАЗАТЕЛЬСТво. $(\Longrightarrow)$ Из включения $\Pi_{q, p_{2}} \subset \Pi_{q, p_{1}}$ при $p_{1} \leqslant p_{2}$ сразу следует $(q, 1)$-абсолютная суммируемость оператора $I\left(L^{\infty}, X\right)$, которая эквивалентна неравенству

$$
\left(\sum\left\|x_{i}\right\|_{X}^{q}\right)^{1 / q} \leqslant \pi_{q, 1}(I)\left\|\sum\left|x_{i}\right|\right\|_{L^{\infty}}
$$

справедливому для произвольного конечного набора векторов $\left\{x_{1}, x_{2}, \ldots, x_{n}\right\} \subset L^{\infty}$. Полагая $x_{i}=\varkappa_{[(i-1) / n, i / n]}, i=1, \ldots, n$, мы приходим к неравенству $\varphi_{X}(t) \leqslant \pi_{q, 1}(I) t^{1 / q}$, $0 \leqslant t \leqslant 1$, которое ведет к включению $X \supseteq L^{q, 1}$.

$(\Longleftarrow)$ Покажем, что $I\left(L^{\infty}, L^{q, 1}\right) \in \Pi_{q, 1}$. Учитьвая следствие из теоремы Ж. Пизье $[8,2.1 .2]$, этого достаточно для завершения доказательства.

Пусть $\left\{x_{1}, \ldots, x_{n}\right\}$ - произвольньй конечный набор векторов из $L^{\infty}$. Имеем

$$
\begin{aligned}
\sum_{i=1}^{n}\left\|x_{i}\right\|_{q, 1}^{q} & =\sum_{i=1}^{n}\left(\int_{0}^{1}\left(\operatorname{meas}\left(\left|x_{i}\right|>t\right)\right)^{1 / q} d t\right)^{q} \leqslant \sum_{i=1}^{n} \int_{0}^{1} \operatorname{meas}\left(\left|x_{i}\right|>t\right) d t \\
& =\sum_{i=1}^{n} \int_{0}^{1}\left|x_{i}\right| d t \leqslant\left\|\sum_{i=1}^{n}\left|x_{i}\right|\right\|_{L^{\infty}} \cdot
\end{aligned}
$$

Полученное неравенство означает $(q, 1)$-суммируемость оператора $I\left(L^{\infty}, L^{q, 1}\right)$.

Теоремы, доказанные в этом пункте, дают основание для следующего предположения (доказать, которое автору не удалось): если $U-$ некоторый операторный идеал, то существует БСП $X_{U}$ такое, что

$$
I\left(L^{\infty}, X\right) \in U \Longleftrightarrow X \supset X_{U}, X-\mathrm{БC \Pi}
$$

включение справа может быть строгим или нестрогим в зависимости от идеала $U$. Пространство $X_{U}$ естественно назьвать граничным для идеала $U$.

3. Оператор вложения $I\left(X, L^{1}\right)$ для БСП $X$. Каждое БСП $X$ (на $\left.[0,1]\right)$ непрерьвно вложено в $L^{1}[1]$. Оператор $I\left(X, L^{1}\right)$ не является, вообще говоря, строго сингулярным. Хорошо известны примеры бесконечномерных подпространств в $L^{1}$, которые являются подпространствами и в более узких СП. Это так называемые "сквозные" подпространства, о которых подробнее будет сказано ниже.

Тем не менее оказалось, что есть содержательное расширение свойства строгой сингулярности, и оператор $I\left(X, L^{1}\right)$ этим (расширенньм) свойством обладает.

Фиксируем $\varepsilon>0$ и для $f \in X$ определим

$$
M_{f, \varepsilon}=\left\{t \in[0,1]:|f(t)| \geqslant \varepsilon\|f\|_{X}\right\} .
$$

Далее, положим

$$
M_{\varepsilon}^{X}=\left\{f \in X:\left|M_{f, \varepsilon}\right| \geqslant \varepsilon\right\}
$$

Классы $M_{\varepsilon}^{X}$ появились впервые в [10] для $X=L^{p}$ и в [11] для общего СП $X . \mathrm{B}[11]$ были сформулированы и следующие две леммы.

Лемма 3.1. Пусть $K$-подмнохсество БСП $X, X \neq L^{1}$. Нормы пространств $X u L^{1}$ әквивалентны на $K \Longleftrightarrow$ существует $\varepsilon>0$ такое, что $K \subset M_{\varepsilon}^{X}$. 
ДоказАТЕЛЬСтво. $(\Longrightarrow)$ Предположим, что нормы $X$ и $L^{1}$ эквивалентны на $K$ : $\delta\|f\|_{X} \leqslant\|f\|_{1}$ для каждого $f \in K$ и для некоторого $\delta>0$. Предположим, далее, что для любого $\varepsilon>0$ найдется $f_{\varepsilon} \in K$ такая, что $f_{\varepsilon} \notin \boldsymbol{M}_{\varepsilon}^{X}$. Из условия леммы следует, что $X^{\prime} \neq L^{\infty}$, следовательно,

$$
\lim _{t \rightarrow 0} \varphi_{X^{\prime}}(t)=0
$$

(см. [12, лемма 3]). Определим $\varepsilon>0$ так, чтобы $\varphi_{X^{\prime}}(\varepsilon)+\varepsilon \leqslant \delta / 2$. Тогда

$$
\begin{aligned}
\delta\left\|f_{\varepsilon}\right\|_{X} & \leqslant\left\|f_{\varepsilon}\right\|_{1}=\int_{M_{f, \varepsilon}}\left|f_{\varepsilon}(t)\right| d t+\int_{[0,1]-M_{f, \varepsilon}}\left|f_{\varepsilon}(t)\right| d t \\
& \leqslant\left\|f_{\varepsilon}\right\|_{X} \varphi_{X^{\prime}}(\varepsilon)+\varepsilon\left\|f_{\varepsilon}\right\|_{X} \leqslant \frac{\delta}{2}\left\|f_{\varepsilon}\right\|_{X},
\end{aligned}
$$

что невозможно. Следовательно, $K \subset \boldsymbol{M}_{\varepsilon}^{X}$ для некоторого $\varepsilon>0$.

$(\Longleftarrow)$ Пусть $K \subset \boldsymbol{M}_{\varepsilon}^{X}$ для некоторого $\varepsilon>0$. Тогда

$$
\|f\|_{1} \geqslant \int_{M_{f, \varepsilon}}|f(t)| d t \geqslant \varepsilon\|f\|_{X} \cdot\left|M_{f, \varepsilon}\right| \geqslant \varepsilon^{2}\|f\|_{X}
$$

для каждого $f \in K$.

Теперь мы введем, следуя [13], ослабленное свойство строгой сингулярности, о котором говорили в начале пункта.

Пусть $X$ - квазибанахова решетка, $Y$ - банахово или квазибанахово пространство. Оператор $T: X \rightarrow Y$ называется дизбюнктно строго сингулярным (запись $T \in \mathrm{DSS}$ ), если не существует последовательности ненулевых дизъюнктных векторов $\left(x_{n}\right)$ в $X$ такой, что сужение $T$ на подпространство $\operatorname{span}\left(x_{n}\right)$ является изоморфизмом.

Ясно, что каждый строго сингулярный оператор является DSS-оператором. Обратное, вообще говоря, неверно: оператор $I\left(L^{p}, L^{q}\right) \in \mathrm{DSS}$ для $1 \leqslant q<p$, но не является строго сингулярным. "Сквозньгм" подпространством в этой, как и во многих других ситуациях, оказывается подпространство $\overline{\operatorname{span}}^{1}\left(r_{i}\right)$, где $\left(r_{i}\right)_{i=1}^{\infty}$ - функции Радемахера.

Teорема 3.2. Пусть $X-$ БСП, $X \neq L^{1}$. Тогда $I\left(X, L^{1}\right) \in$ DSS.

ДокАЗАТЕЛЬСтво. Предположим, что утверждение теоремы неверно: существует последовательность дизъюнктных функций $\left(f_{n}\right)$ в $X$ такая, что $\left.I\right|_{\operatorname{span}\left(f_{n}\right)}-$ изоморфизм. По лемме 3.1 найдется $\varepsilon>0$ такое, что $\operatorname{span}\left(f_{n}\right) \subset \boldsymbol{M}_{\varepsilon}^{X}$. Множества $M_{f_{n}, \varepsilon}$ дизъюнктны, поэтому получаем

$$
1=|[0,1]| \geqslant\left|\bigcup_{n} M_{f_{n}, \varepsilon}\right|=\sum_{n}\left|M_{f_{n}, \varepsilon}\right|=\infty
$$

так как $\left|M_{f_{n}, \varepsilon}\right| \geqslant \varepsilon$.

Другой способ доказательства теоремы 3.2 был недавно предложен С. В. Асташкиным.

Следует заметить, что теорема 3.2 в определенном смысле характеризует именно пространство $L^{1}$. В работе [13] было показано, что для любого пространства $L^{p}$ с $p>1$ сушествует БСП $X \subset L^{p}$ такое, что $I\left(X, L^{p}\right) \notin$ DSS. 
4. Операторы вложения квазибанаховых симметричных пространств на $[0,1]$. Сквозные подпространства. Каждое КБСП $X$ можно считать $\gamma$-банаховым для некоторого $\gamma \in(0,1)$, т.е. в дополнение к аксиомам квазинормы можно добавить неравенство

$$
\|f+g\|^{\gamma} \leqslant\|f\|^{\gamma}+\|g\|^{\gamma}, \quad f, g \in X
$$

(см. $[6,6.2 .5])$.

В [14] показано, что для $\gamma$-банахова пространства $X$ справедливы непрерывные включения

$$
L^{\infty} \subset X \subset L^{\gamma, \infty}
$$

где

$$
L^{\gamma, \infty}=\left\{f:\|f\|_{\gamma, \infty}=\sup _{t>0} t^{1 / \gamma} f^{*}(t)<\infty\right\}
$$

Первое из них уже отмечалось выше (см. лемму 2.1), доказательство второго приведем для удобства в виде следующей леммы.

Лемма 4.1. Пусть $X-\gamma$-банахово пространство, $0<\gamma<1$. Тогда $X \subset L^{\gamma, \infty}$ $u\left\|I\left(X, L^{\gamma, \infty}\right)\right\| \leqslant 2^{1 / \gamma}$.

ДокаЗАТЕЛЬСтво. Для $t \in[0,1]$ имеет место неравенство

$$
\varphi_{X}(t) \geqslant\left(\frac{t}{2}\right)^{1 / \gamma}
$$

Действительно, пусть $t \in[1 /(n+1), 1 / n]$ для некоторого $n \in \mathbb{N}$. Тогда

$$
\begin{aligned}
1=\varphi_{X}^{\gamma}(1)=\left\|\varkappa_{[0,1]}\right\|_{X}^{\gamma} & \leqslant \sum_{k=1}^{n+1}\left\|\varkappa_{[(k-1) /(n+1), k /(n+1)]}\right\|_{X}^{\gamma} \\
& =(n+1) \varphi_{X}^{\gamma}\left(\frac{1}{n+1}\right) \leqslant(n+1) \varphi_{X}^{\gamma}(t) \\
& =\frac{n+1}{n} \cdot n \varphi_{X}^{\gamma}(t) \leqslant 2 \frac{1}{t} \varphi_{X}^{\gamma}(t) .
\end{aligned}
$$

Отсюда

$$
\|f\|_{X}=\left\|f^{*}\right\|_{X} \geqslant\left\|f^{*} \varkappa_{[0, t]}\right\|_{X} \geqslant f^{*}(t) \varphi_{X}(t) \geqslant f^{*}(t)\left(\frac{t}{2}\right)^{1 / \gamma}, \quad t \in[0,1],
$$

и

$$
\|f\|_{\gamma, \infty} \leqslant 2^{1 / \gamma}\|f\|_{X}, \quad f \in X
$$

Пространство $L^{\gamma, \infty}$ является $\gamma$-банаховым пространством. Это легко заметить, если ввести функционал

$$
\|f \mid\|=\inf \left\{\left(\sum_{i=1}^{n}\left\|f_{i}\right\|_{\gamma, \infty}^{\gamma}\right)^{1 / \gamma}: f=\sum_{i=1}^{n} f_{i}, f_{i} \in L^{\gamma, \infty}\right\}
$$

Таким образом, $L^{\gamma, \infty}$ - это наибольшее $\gamma$-банахово СП на $[0,1], 0<\gamma<1$. 
Если дополнительно предположить, что $\gamma$-нормированное пространство $X$ является $\gamma$-вьпуклым, т.е. для произвольных $\left\{f_{1}, \ldots, f_{n}\right\} \subset X$

$$
\left\|\left(\sum_{i=1}^{n}\left|f_{i}\right|^{\gamma}\right)^{1 / \gamma}\right\| \leqslant\left(\sum_{i=1}^{n}\left\|f_{i}\right\|^{\gamma}\right)^{1 / \gamma}
$$

то включения (2) уточняются следующим образом:

$$
L^{\infty} \subset X \subset L^{\gamma}
$$

т.е. наибольшим $\gamma$-вьпукльм $\gamma$-БСП оказьвается пространство $L^{\gamma}$, более узкое, чем $L^{\gamma, \infty}$. Второе из этих включений обосновьвается по схеме доказательства теоремы 4.1 в [1].

Теорема 4.2. Пусть $X-\gamma$-банахово и $\gamma$-выпуклое СП на $[0,1]$. Тогда $I\left(L^{\infty}, X\right)$ строго сингулярен и $I\left(X, L^{\gamma}\right) \in \mathrm{DSS}$.

ДокАЗАТЕЛьСТво. В основе доказательства лежит конструкция вспомогательного пространства $X_{\gamma}:=\left\{f:|f(t)|^{1 / \gamma} \in X\right\}$. На этом пространстве определим функционал $\|f\|_{(\gamma)}=\left\||f|^{1 / \gamma}\right\|_{X}^{\gamma}$, который оказьвается, как легко проверить с учетом $\gamma$-вьпуклости, нормой. Таким образом, в заданной условием теоремы ситуации существует банахово СП $X_{\gamma}$ такое, что $X_{\gamma} \subset X$. (Заметим, что если $X=L^{\gamma}$, то $X_{\gamma}=L^{1}$.) Теперь строгая сингулярность оператора $I\left(L^{\infty}, X\right)$ может быть обоснована теоремой 2.6. Мы факторизуем этот оператор через БСП $X_{\gamma}$. Оператор $I\left(X, L^{\gamma}\right) \in \mathrm{DSS}$, так как $I\left(X_{\gamma}, L^{1}\right) \in \operatorname{DSS}$ (теорема 3.3 ), и остается заметить

$$
I(X, Y) \in \mathrm{DSS} \Longleftrightarrow I\left(X_{\gamma}, Y_{\gamma}\right) \in \mathrm{DSS}
$$

Последняя эквивалентность следует прямо из определений.

Показать, что оператор $I\left(X_{1}, X_{2}\right)$ не является строго сингулярным, значит обнаружить бесконечномерное подпространство $H \subset X_{1}$, которое замкнуто в $X_{2}$. В силу теоремы о замкнутом графике на таком подпространстве (квази-)нормы пространств $X_{1}$ и $X_{2}$ эквивалентны. Мы назвали вьше такое подпространство "сквозным". В западной литературе употребляют термин "strongly embedded" - "сильно вложенные" (см., например, [15]).

По всей видимости, самым известным сквозным подпространством является подпространство $R_{2}$, порожденное функциями Радемахера $\left(r_{i}\right)_{i=1}^{\infty}$. Его можно описать следующим образом:

$$
R_{2}=\left\{f \in L_{0}: f={ }^{L_{0}} \sum c_{k} r_{k},\left(c_{k}\right) \in \ell^{2}\right\}
$$

В [12] показано, что $R_{2}$ является сквозным подпространством для БСП $X$ таких, что $X \supset G$, где $G$ - замыкание $C[0,1]$ (или $L^{\infty}$ ) по норме пространства Орлича $L_{M_{2}}$, где $M_{2}(u)=e^{u^{2}}-1$. Так как $R_{2}$ замкнуто и в $L_{0}$ (обосновать это можно, например, $\mathrm{c}$ помощью теоремы А.Н. Колмогорова о трех рядах [16]), то получается, что $R_{2}$ является сквозным подпространством и в более широком диапазоне СП $X$, в том числе и в квазибанаховых: $G \subset X \subset L_{0}$.

Приведем еще несколько примеров сквозных подпространств. Их общей особенностью является то, что они порождены последовательностью независимых симметричных 
(т.е. функции $f$ и $-f$ равноизмеримы) одинаково распределенных функций (или случайных величин).

Вешественная функция $s^{(r)}(\omega), 0 \leqslant \omega \leqslant 1,0<r \leqslant 2$, назьвается $r$-устойчивой, если

$$
\int_{0}^{1} e^{i t s^{(r)}(\omega)} d \omega=e^{-c|t|^{r}}
$$

для некоторого $c>0$ и для всех $t \in \mathbb{R}$. Такая функция по определению симметрична. 2 -устойчивая функция - это хорошо известная гауссовская случайная величина. Наряду с $r$-устойчивыми функциями рассмотрим симметричную функцию, для которой

$$
\left(g^{(r)}(\omega)\right)^{*}=\omega^{-1 / r}, \quad 0<r<\infty, \quad 0<\omega \leqslant 1 .
$$

TEорема 4.3. Пусть $0<r<2,\left(s_{n}^{(r)}\right),\left(g_{n}^{(r)}\right)$ - независимые копии функций $s^{(r)} u$ $g^{(r)}$ соответственно. Тогда $\overline{\operatorname{span}}^{L}\left(s_{n}^{(r)}\right) u \overline{\operatorname{span}}^{L}\left(g_{n}^{(r)}\right)$ - сквозные подпространства для СП $X$ таких, что $X \supseteq L^{r, \infty}$, причем

$$
\left\|\sum a_{n} s_{n}^{(r)}\right\|_{X} \approx\left\|\sum a_{n} g_{n}^{(r)}\right\|_{X} \approx\left(\sum\left|a_{n}\right|^{r}\right)^{1 / r} \text {. }
$$

ДокАЗАтЕльство. Во-первых, заметим, что пространство $L^{r, \infty}$ является естественной границей для рассматриваемых подпространств, так как

$$
\begin{gathered}
d\left(s^{(r)} ; t\right)=\operatorname{meas}\left(\omega:\left|s^{(r)}\right|>t\right) \sim \frac{1}{t^{r}}, \quad t \rightarrow \infty, \\
d\left(g^{(r)} ; t\right)=\frac{1}{t^{r}}, \quad t \geqslant 1,
\end{gathered}
$$

а это означает, что

$$
s^{(r)} \in X \Longleftrightarrow X \supseteq L^{r, \infty},
$$

аналогично для $g^{(r)}$.

Оценки снизу для $\left\|\sum a_{n} s_{n}^{(r)}\right\|_{X}$ и $\left\|\sum a_{n} g_{n}^{(r)}\right\|_{X}$ следуют из теоремы Колмогорова о трех рядах [16], которая позволяет получить критерии сходимости случайных рядов в $L_{0}$.

Прежде чем получить оценку сверху, докажем несколько вспомогательных предложений.

ЛЕмма 4.4. Если $\left(\xi_{n}\right)$ - независимые симметричные случайные величины в пространстве $L^{r, \infty}, 0<r<\infty$, то

$$
\left\|\sum \xi_{n}\right\|_{L^{r, \infty}} \approx\left\|\left(\sum\left|\xi_{n}\right|^{2}\right)^{1 / 2}\right\|_{L^{r, \infty}}
$$

т.е. сущ,ствуют константы $C_{1}, C_{2}>0$ такие, что для любого $N$

$$
C_{1}\left\|\left(\sum_{n=1}^{N}\left|\xi_{n}\right|^{2}\right)^{1 / 2}\right\|_{L^{r, \infty}} \leqslant\left\|\sum_{n=1}^{N} \xi_{n}\right\|_{L^{r, \infty}} \leqslant\left\|\left(\sum_{n=1}^{N}\left|\xi_{n}\right|^{2}\right)^{1 / 2}\right\|_{L^{r, \infty}} .
$$

(В дальнейшем знак ‘ $\approx$ всегда будет использоваться именно в таком смысле). 
ДокАЗАТЕЛЬСТво. Последовательности $\left(\xi_{n}(\omega)\right)$ и $\left(\xi_{n}(\omega) r_{n}(t)\right)$, где $r_{n}(t)$ - функции Радемахера, одинаково распределены, или подобны в вероятностном смысле (см., например, $[17,5.2 .1])$. Следовательно,

$$
\left\|\sum_{n=1}^{N} \xi_{n}\right\|_{L^{r, \infty}}=\left\|\sum_{n=1}^{N} \xi_{n} r_{n}\right\|_{L^{r, \infty}\left([0,1]^{2}\right)} \approx\left\|\left(\sum_{n=1}^{N}\left|\xi_{n}\right|^{2}\right)^{1 / 2}\right\|_{L^{r, \infty}},
$$

последняя эквивалентность доказана, например, в [18, 2.d.1].

Пусть $\left\{f_{1}, \ldots, f_{n}\right\} \subset L^{r, \infty}[0,1]$. Обозначим через

$$
\sum_{k=1}^{n} \oplus f_{k}:=F \in L^{r, \infty}[0, \infty)
$$

функцию, для которой

$$
d(F ; \cdot)=\sum_{k=1}^{n} d\left(f_{k} ; \cdot\right)
$$

В качестве $F$ можно взять, например, функцию

$$
F(t)=\sum_{k=1}^{n} f_{k}(t-k+1) \varkappa_{[k-1, k)}(t), \quad 0 \leqslant t \leqslant n .
$$

Лемма 4.5 [15]. Пусть $0<r<\infty, r \neq 2$, существует константа $C>0$ такая, что для любых $\left\{f_{1}, \ldots, f_{N}\right\} \subset L^{r, \infty}$ справедливы неравенства

$$
\begin{array}{ll}
\left\|\left(\sum_{n=1}^{N}\left|\xi_{n}\right|^{2}\right)^{1 / 2}\right\|_{L^{r, \infty}} \leqslant C\left\|\sum_{n=1}^{N} \oplus f_{n}\right\|_{L^{r, \infty}[0, \infty)}, & 0<r<2, \\
\left\|\sum_{n=1}^{N} \oplus f_{n}\right\|_{L^{r, \infty}[0, \infty)} \leqslant C\left\|\left(\sum_{n=1}^{N}\left|\xi_{n}\right|^{2}\right)^{1 / 2}\right\|_{L^{r, \infty}}, & 2<r<\infty .
\end{array}
$$

Лемма 4.6. Пространство $L^{r, \infty}(I), I=[0,1]$ или $[0, \infty)$, удовлетворяет верхней г-оценке для дизбюнктных функиий, т.е.

$$
\left\|\sum_{k=1}^{n} f_{k}\right\| \leqslant\left(\sum_{k=1}^{n}\left\|f_{k}\right\|^{r}\right)^{1 / r}
$$

$\left\{f_{1}, \ldots, f_{n}\right\}-$ произвольный набор попарно дизбюнктных функиий. 
ДокаЗАТЕЛЬСТво. Имеем

$$
\begin{aligned}
\left\|\sum_{k=1}^{n} f_{k}\right\|_{r, \infty}^{r} & =\sup _{t>0} d\left(\sum f_{k} ; t\right) t^{r}=\sup _{t>0} \sum d\left(f_{k} ; t\right) t^{r} \\
& \leqslant \sum_{k=1}^{n} \sup _{t>0} t^{r} d\left(f_{k} ; t\right)=\sum\left\|f_{k}\right\|_{r, \infty}^{r} .
\end{aligned}
$$

Продолжим доказательство теоремы 4.3.

Оценим $\left\|\sum a_{n} g_{n}^{(r)}\right\|_{X}$ сверху, используя леммы 4.4-4.6:

$$
\begin{aligned}
\left\|\sum a_{n} g_{n}^{(r)}\right\|_{X} & \leqslant C_{1}\left\|\sum a_{n} g_{n}^{(r)}\right\|_{L^{r, \infty}} \approx C_{2}\left\|\left(\sum\left|a_{n} g_{n}^{(r)}\right|^{2}\right)^{1 / 2}\right\|_{L^{r, \infty}} \\
& \leqslant C_{3}\left\|\sum \oplus a_{n} g_{n}^{(r)}\right\|_{L^{r, \infty}[0, \infty)} \leqslant C_{4}\left(\sum\left|a_{n}\right|^{r}\right)^{1 / r} .
\end{aligned}
$$

Сумма $\left\|\sum a_{n} s_{n}^{(r)}\right\|_{X}$ оценивается аналогично.

Таким образом, если $0<r<2$, то последовательности $\left(s_{n}^{(r)}\right)$ и $\left(g_{n}^{(r)}\right)$ порождают сквозные подпространства, изоморфные $\ell^{r}$ и имеюшие одинаковые границы.

Для $r \geqslant 2$ отмеченное сходство отсутствует. Действительно, само понятие $r$-устойчивой функции не имеет смысла при $r>2$. Однако, для $\left(g_{n}^{(r)}\right)$ имеет место

ТЕОРема 4.7. Пусть $r>2,\left(g_{n}^{(r)}\right)$ - независимые копии функиии $g^{(r)}$. Тогда $\overline{\operatorname{span}}^{L_{0}}\left(g_{n}^{(r)}\right)$ - сквозное подпространство для СП $X$ таких, что $X \supseteq L^{r, \infty}, n p u^{-}$ чем

$$
\left\|\sum a_{n} g_{n}^{(r)}\right\|_{X} \approx\left(\sum\left|a_{n}\right|^{2}\right)^{1 / 2}
$$

m.e. $\overline{\operatorname{span}}^{L}\left(g_{n}^{(r)}\right) \approx \ell^{2}$.

ДокАЗАТЕЛЬСтво. Нижняя оценка получается из теоремы о трех рядах. Для того чтобы получить оценку сверху, заметим, что пространство $L^{r, \infty}$ при $r>2$ является 2-вьпуклым. Этот факт легко получить из [19], если учесть, что $L^{r, \infty}=\boldsymbol{M}\left(\varphi_{r^{\prime}}\right)$, $\varphi_{r^{\prime}}(t)=t^{1 / r^{\prime}}, 1 / r^{\prime}+1 / r=1$, в обозначениях [19].

Итак, согласно лемме 4.4 имеем

$$
\left\|\sum a_{n} g_{n}^{(r)}\right\|_{X} \leqslant C_{1}\left\|a_{n} g_{n}^{(r)}\right\|_{L^{r, \infty}} \approx C_{2}\left\|\left(\sum\left|a_{n} g_{n}^{(r)}\right|^{2}\right)^{1 / 2}\right\|_{L^{r, \infty}} \leqslant C_{3}\left(\sum\left|a_{n}\right|^{2}\right)^{1 / 2} .
$$

В доказанной теореме важно, что $r>2$. Пространство $L^{2, \infty}$ не является 2-выпуклым. Это видно уже из включения $L^{2} \subset L^{2, \infty}$, так как любое $\gamma$-выпуклое СП $X$ удовлетворяет условию $X \subset L^{\gamma}, 0<\gamma<\infty[18]$. Оставшийся пока неразобранным случай $r=2$ описывается следующей теоремой. 
TЕорема 4.8. 1) Пусть $\left(s_{n}^{(2)}\right)$ - независимые копии гауссовской случайной величины $s^{(2)}$. Тогда $\overline{\mathrm{span}}^{L_{0}}\left(s_{n}^{(2)}\right) \approx \ell^{2}$ и является сквозным подпространством для СП $X, X \supseteq L_{M_{2}}$, әде $L_{M_{2}}$ - пространство Орлича, построенное по функиии $M_{2}=e^{u^{2}}-1$.

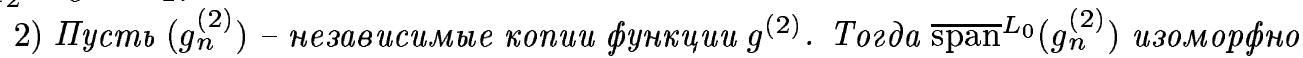
пространству Орлича последовательностей $\ell_{N}$, где $N(u)=u^{2} \ln (1+1 /|u|), u \neq 0$, и является сквозным подпространством для СП $X, X \supseteq L^{2, \infty}$.

ДокАЗАТЕЛЬСТво. 1) Эта часть теоремы может считаться классической, и включена лишш для полноты изложения (подробнее см. [17, гл. 5]). При этом следует учесть, что

$$
f \in L_{M_{2}} \Longleftrightarrow \int_{0}^{1} M_{2}(\lambda|f(t)|) d t<\infty
$$

для некоторого $\lambda>0$. Есть и другое описание пространства $L_{M_{2}}$ : оно состоит из функций, которые мажорируются функцией, равноизмеримой с некоторым растяжением функции $\left|s^{(2)}\right|+1[18,2 . \mathrm{b} .4]$.

2) Нижняя оценка вновь следует из теоремы о трех рядах. Согласно этой теореме сходимость ряда $\sum a_{n} g_{n}^{(2)}$ в $L_{0}$ эквивалентна сходимости трех числовых рядов:

1) $\sum \mathrm{P}\left(\left|a_{n} g_{n}^{(2)}\right|>1\right)$

2) $\sum \mathrm{E}\left(a_{n} g_{n}^{(2)}\right)^{\prime}$

3) $\sum \mathrm{D}\left(a_{n} g_{n}^{(2)}\right)^{\prime}$;

где $\mathrm{E}$ и $\mathrm{D}$ - математическое ожидание и дисперсия соответственно, $\left(\xi_{n}\right)^{\prime}=\xi_{n} \cdot \varkappa_{\left[\left|\xi_{n}\right| \leqslant 1\right]}$. Сходимость ряда 1) эквивалентна соотношению $\left(a_{n}\right) \in \ell^{2}$, сходимость ряда 2) обеспечивается симметрией функции $g^{(2)}$, для выяснения условия сходимости ряда 3 ) заметим, что

$$
\mathrm{D}\left(a_{n} g_{n}^{(2)}\right)^{\prime}=\mathrm{E}\left[\left(a_{n} g_{n}^{(2)}\right)^{\prime}\right]^{2}=2 a_{n}^{2} \int_{1}^{1 /\left|a_{n}\right|} t^{-1} d t=2 a_{n}^{2}|\ln | a_{n}|| \sim 2 a_{n}^{2} \ln \left(1+\frac{1}{\left|a_{n}\right|}\right)
$$

Для доказательства верхней оценки нам потребуется следующая лемма.

ЛЕмма 4.9. Пусть $e_{j}(\omega), j=1,2, \ldots,-$ последовательность неотрицательных равноизмеримых функиий, причем $d\left(e_{j} ; t\right)=1 / t, t \geqslant 1$. Если $\alpha_{j}>0, j=1,2, \ldots, u$ $\sum \alpha_{j} \ln \left(1+1 / \alpha_{j}\right)<\infty$, то $\sum \alpha_{j} e_{j}$ сходится в пространстве $L^{1, \infty}$.

ДокАЗАТЕЛЬСтво. Функция $\psi(u)=u \ln (1+1 / u), u>0$, квазивогнута $[1$, с. 70$]$ и, значит, полуаддитивна. Пусть $\sum \psi\left(\alpha_{k}\right)=a$, тогда $\psi\left(\sum \alpha_{k}\right) \leqslant a$ и $\sum \alpha_{k} \leqslant \psi^{-1}(a)$. Пусть $\Delta$ - измеримое подмножество $[0,1]$ и $|\Delta|=t>0$. Тогда

$$
t \inf _{\omega \in \Delta} \sum_{j=1}^{n} \alpha_{j} e_{j}(\omega) \leqslant \int_{\Delta}\left(\sum_{j=1}^{n} \alpha_{j} e_{j}(\omega)\right) d \omega
$$

Обозначим через

$$
\Delta_{j}=\left\{\omega \in \Delta: e_{j}(\omega)>\frac{2 \psi^{-1}(a)}{\alpha_{j} t}\right\}, \quad j=1,2, \ldots
$$


Заметим, что

$$
\left|\Delta_{j}\right| \leqslant\left|\left\{\omega: e_{j}(\omega)>\frac{2 \psi^{-1}(a)}{\alpha_{j} t}\right\}\right|=\frac{\alpha_{j} t}{2 \psi^{-1}(a)}, \quad j=1,2, \ldots,
$$

поэтому

$$
\left|\Delta-\bigcup_{j=1}^{n} \Delta_{j}\right| \geqslant t-\frac{t}{2 \psi^{-1}(a)} \sum_{j=1}^{n} \alpha_{j} \geqslant t-\frac{t}{2 \psi^{-1}(a)} \psi^{-1}(a)=\frac{t}{2}, \quad n=1,2, \ldots .
$$

Рассмотрим теперь

$$
\begin{aligned}
\inf _{\omega \in \Delta} \sum_{j=1}^{n} \alpha_{j} e_{j}(\omega) & \leqslant \inf _{\Delta-\bigcup_{j=1}^{n} \Delta_{j}} \sum_{j=1}^{n} \alpha_{j} e_{j} \\
& \leqslant \frac{1}{m\left(\Delta-\bigcup_{j=1}^{n} \Delta_{j}\right)} \int_{\Delta-\cup_{j=1}^{n} \Delta_{j}}\left(\sum_{j=1}^{n} \alpha_{j} e_{j}(\omega)\right) d \omega \\
& \leqslant \frac{2}{t} \sum_{j=1}^{n} \alpha_{j} \int_{\Delta-\cup_{j=1}^{n} \Delta_{j}} e_{j} d \omega \leqslant \frac{2}{t} \sum_{j=1}^{n} \alpha_{j} \int_{\alpha_{j} t /\left(2 \psi^{-1}(a)\right)}^{\alpha_{j} t /\left(2 \psi^{-1}(a)\right)+t} e_{j}^{*}(s) d s \\
& =\frac{2}{t} \sum_{j=1}^{n} \alpha_{j} \int_{\alpha_{j} t /\left(2 \psi^{-1}(a)\right)}^{\alpha_{j} t /\left(2 \psi^{-1}(a)\right)+t} \frac{d s}{s} \\
& \leqslant \frac{2}{t}\left(1+2 \psi^{-1}(a)\right) \sum_{j=1}^{n} \alpha_{j} \ln \left(1+\frac{1}{\alpha_{j}}\right) \leqslant \frac{2 a}{t}\left(1+2 \psi^{-1}(a)\right), \quad t>0 .
\end{aligned}
$$

Таким образом,

$$
\inf _{\omega \in \Delta,|\Delta|=t} \sum_{j=1}^{n} \alpha_{j} e_{j}(\omega) \leqslant \frac{2 a}{t}\left(1+2 \psi^{-1}(a)\right), \quad t>0, \quad n=1,2, \ldots,
$$

и, следовательно,

$$
\begin{aligned}
\left(\sum_{j=1}^{n} \alpha_{j} e_{j}\right)^{*}(t) & =\inf \left\{\tau:\left|\left\{\omega: \sum_{j=1}^{n} \alpha_{j} e_{j}(\omega)>\tau\right\}\right|<t\right\} \\
& \leqslant \frac{2 a}{t}\left(1+2 \psi^{-1}(a)\right), \quad t>0, \quad n=1,2, \ldots
\end{aligned}
$$

или

$$
\left\|\sum_{j=1}^{n} \alpha_{j} e_{j}\right\|_{1, \infty} \leqslant 2 a\left(1+2 \psi^{-1}(a)\right), \quad n=1,2, \ldots .
$$

Продолжим доказательство теоремы 4.8 .

Пусть $a=\left(a_{k}\right)_{k=1}^{\infty} \in \ell_{N}$, т.е.

$$
\sum_{k=1}^{\infty} a_{k}^{2} \ln \left(1+\frac{1}{a_{k}^{2}}\right)<\infty
$$


(функции $N(u)=u^{2} \ln (1+1 / u)$ и $N_{1}(u)=u^{2} \ln \left(1+1 / u^{2}\right)$ эквивалентны в нуле), тогда $\left(a_{k}^{2}\right)_{k=1}^{\infty}$ удовлетворяет условиям леммы 4.9 , согласно которой ряд

$$
\sum_{k=1}^{\infty} a_{k}^{2}\left(g_{k}^{(2)}\right)^{2}
$$

сходится в $L^{1, \infty}$. Но

$$
\left\|\sum a_{k} g_{k}^{(2)}\right\|_{2, \infty}^{2} \approx\left\|\left(\sum\left|a_{k} g_{k}^{(2)}\right|^{2}\right)^{1 / 2}\right\|_{2, \infty}^{2}=\left\|\sum a_{k}^{2}\left(g_{k}^{(2)}\right)^{2}\right\|_{1, \infty},
$$

поэтому ряд $\sum a_{k} g_{k}^{(2)}$ сходится в $L^{2, \infty}$.

\section{СПИСОК ЦИТИРОВАННОЙ ЛИТЕРАТУРЫ}

[1] Крейн С. Г., Петунин Ю. И., Семёнов Е. М. Интерполяция линейных операторов. M.: Наука, 1978.

[2] Лозановский Г.Я. О некоторых банаховых структурах // Сиб. матем. ж. 1969. Т. 10. № 3. C. 584-599.

[3] Бухвалов А. В., Векслер А. И., Лозановский Г.Я. Банаховы решетки -некоторые банаховы аспекты теории // УМН. 1979. Т. 34. № 2. С. 136-183.

[4] Эдвардс Р. Функциональный анализ. М.: Мир, 1969.

[5] Simons S. On the Dunford-Pettis property // Math. Ann. 1975. V. 216. № 3. P. 225-231.

[6] Пич А. Операторные идеалы. М.: Мир, 1982.

[7] Рудин У. Функциональный анализ. М.: Мир, 1975.

[8] Кисляков С. В. Абсолютно суммирующие операторынадиск-алгебре // Алгебраи анализ. 1991. T. 3. № 4. C. 1-77.

[9] Макаров Б. М. Абсолютно суммирующие операторы и некоторые их приложения // Алгебра и анализ. 1991. Т. 3. №2. С. 1-76.

[10] Kadec M. I., Pelczynski A. Bases, lacunary sequences and complemented subspaces in the space $L^{p} / /$ Studia Math. 1962. V. 21. № 2. P. 161-176.

[11] Новиков С.Я., Семёнов Е.М., Токарев Е.В. Структура подпространств пространств $\Lambda_{p}(\varphi) / /$ Докл. АН СССР. 1979. Т. 247. № 3. С. 552-554.

[12] Rodin V. A., Semyonov E. M. Rademacher series in symmetric spaces // Anal. Math. 1975. V. 1. № 2. P. 161-176.

[13] Hernandez F. L., Rodrigues-Salinas B. On $\ell^{p}$-complemented copies in Orlicz spaces. II // Israel J. Math. 1989. V. 68. P. 27-55.

[14] Bastero J., Hudzik H., Steinberg A. On smallest and largest spaces among RI $p$-Banach function spaces $(0<p<1)$ // Indag. Math. (N.S.) 1991. V. 2. № 3. P. 283-288.

[15] Carothers N.L., Dilworth S.J. Geometry of Lorentz Spaces via Interpolation // Longhorn Notes. Funct. Anal. Seminar: The Univ. of Texas at Austin, 1985-86. P. 107-133.

[16] Ширяев А. Н. Вероятность. М.: Наука, 1989.

[17] Вахания Н. Н., Тариеладзе В. И., Чобанян С. А. Вероятностные распределения в банаховых пространствах. М.: Наука, 1985.

[18] Lindenstrauss J., Zafriri L. Classical Banach Spaces. II. Berlin: Springer, 1979.

[19] Новиков С.Я. Котип и тип функциональных пространств Лоренца // Матем. заметки. 1982. T. 32. № 2. C. 213-221. 